\title{
Wie die Politik das Rationieren an den Arzt delegieren möchte
}

Jürg Schlup

Dr. med., Präsident der FMH

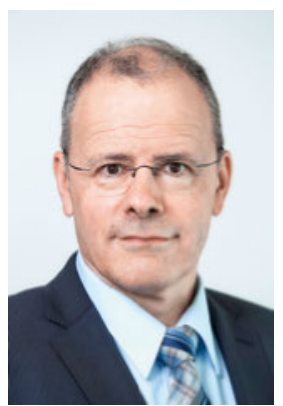

Erst vor wenigen Monaten schnitt unser Gesundheitssystem in einem internationalen Ranking erneut als eines der besten ab: In Bezug auf Qualität und Zugänglichkeit der Versorgung erreichte die Schweiz unter 195 Ländern den dritten Platz [1] $]^{1}$. Die Krankenkassenprämien, die der Schweizer Durchschnittshaushalt für diesen allgemeinen Zugang zu hochqualitativen Behandlungen bezahlt, betragen 5,8\% seines Bruttoeinkommens [2]. Und obwohl Schweizer Haushalte wachsende Beträge für die Gesundheit aufwenden müssen, bleibt ihnen nach dem Bezahlen der Prämien immer mehr Geld für Konsum und Ersparnisse übrig [3, S.10]. Mehr als drei von vier Stimmberechtigten sind zufrieden mit dem Gesundheitswesen und erachten Qualität und Quantität der Gesundheitsleistungen für wichtiger als den Preis [4].

Angesichts hoher Zufriedenheit und volkswirtschaftlich gut tragbarer Kosten [3, S.10] sollten prioritär die $11 \%$ der Bevölkerung unterstützt werden, denen die

Steigenden Anforderungen an das Gesundheitswesen soll ein begrenztes Budget gegenübergestellt werden.

Krankenkassenprämien dauerhaft Probleme bereiten [4]. Auch die Entlastung des Prämienzahlers durch die einheitliche Finanzierung ambulanter und stationärer Leistungen sollte prioritär sein, da sich so jährlich milliardenhohe Effizienzpotentiale realisieren liessen [5]. Stattdessen kündigte das EDI bereits 2016 an, einen radikalen Systemwechsel prüfen zu wollen: nämlich Budgets als Steuerungsinstrumente einzusetzen [6]. Insofern überrascht es wenig, dass die zur Unterstützung dieser Arbeiten eingesetzte Expertengruppe nun auch «die Festlegung einer verbindlichen Zielvorgabe für das OKP-Kostenwachstum [...] als übergeordnete Massnahme zur Kostendämpfung im Gesundheitswesen" [7, S.24] vorschlägt. Steigenden Anforderungen an das Gesundheitswesen soll ein begrenztes Budget gegenübergestellt werden. Vorgegebene "Globalziele» würden auf einzelne Versorgungsbereiche verteilt, wobei die Experten ausdrücklich auch «eine Einführung von Globalzie- len ohne Detailkenntnisse des Effizienzsteigerungspotenzials» [5, S.30] vorschlagen. Überschreitungen des politisch vorgegebenen Budgets sollen Ärzte durch weniger Leistungen vermeiden. Geht der Behandlungsbedarf der Patienten jedoch über das Planbudget hinaus, bleibt dem Arzt nur Leistungen vorzuenthalten - oder

Benötigen die Patienten mehr als die Politik vorgibt, muss der Arzt entweder Leistungen vorenthalten oder wird finanziell sanktioniert.

Sanktionen wie "automatische Tarifreduktionen" $[7$, S.29] zu erdulden. Die Therapiefreiheit wird somit zum Loyalitätskonflikt zwischen Plansoll und Patienten. Gemäss Expertenbericht hätte der Patient hingegen keinen Nachteil, da ja ohnehin 20\% der Leistungen unnütz seien: Es gelte «den erforderlichen Druck auf die verschiedenen Akteure auszuüben» [7, S.27], das Effizienzpotential voll auszuschöpfen. "Auch der Blick ins Ausland» zeige, «dass im Interesse der Bevölkerung ein stärkeres Eingreifen erforderlich ist, um die nötigen Effizienzsteigerungen und Einsparungen anzustossen» [7, S.5]. Dies erstaunt angesichts der Zweiklassenmedizin in Deutschland, wo 87\% der Bevölkerung als Grundversicherte von der Budgetierung betroffen sind. In Nordrhein-Westfalen müssen Grundversicherte auf Termine beim Augenarzt oder Radiologen 38 bzw. 42 Tage warten - Privatpatienten nur fünf [8]. Ähnlich zeigte eine Untersuchung der AOK Rheinland/Hamburg, dass Grundversicherte dort 71 Tage auf einen Kardiologentermin warten mussten - 52 Tage länger als Privatversicherte [9].

Es sind somit Zweifel angebracht, ob Eingriffe und Planungsvorgaben eines - für die Umsetzung des Globalbudgets zwangsläufig deutlich aufgerüsteten - Verwaltungsapparats die Gesundheitsversorgung verbessern. Die Budgetierungsvorbilder Niederlande, Frankreich und Deutschland belegen im oben genannten Länderranking deutlich hinter der Schweiz die Plätze 9, 15 und 20. Für diese Gesundheitsversorgung auf Platz 20 bezahlen die deutschen Prämienzahler 14,6\% ihres Bruttolohns an die Krankenversicherung. 


\section{Literatur}

1 GDB 2015 Healthcare Access and Quality Collaborators. Healthcare Access and Quality Index based on mortality from causes amenable to personal health care in 195 countries and territories, 19902015: a novel analysis from the Global Burden of Disease Study. The Lancet. 2015;390(10091):231-66, 15.7.2017.

2 Medienmitteilung BFS, 25.11.2016. Haushaltsbudgeterhebung 2014.

3 Früh M, Gyger P, Reich O (Dezember 2016). Helsana-Report. Ausgabenentwicklungen in der Gesundheitsversorgung; URL: https:// www.helsana.ch/docs/helsana-ausgabenreport-2016.pdf.

4 Bieri U, et al. (2017). Das Wichtigste in Kürze zum Gesundheitsmonitor 2017, GfS Bern.
5 Klaus G. (November 2016) Dank einheitlicher Finanzierung mehr Effizienz. Helsana Standpunkt 4/16; S. 3-5.

6 Medienmitteilung BAG. Mengenwachstum im Gesundheitswesen eindämmen - zusätzliche Massnahmen nötig. Bern, 2.9.2016.

7 Expertenbericht: Kostendämpfungsmassnahmen zur Entlastung der OKP vom 24.8.2017, publiziert am 25.10.2017.

8 Westfalenpost, 21.4.2017; Ulf Meinke. Privatpatienten werden deutlich bevorzugt beim Arzttermin.

9 Spiegel online, 17.7.2011. Ärzte diskriminieren Kassenpatienten systematisch. 